DOI: 10.1002/adom.((please add manuscript number))

Submitted to

Article type: Communication (3000 words, 4 images, 4 journal pages). Currently we have 3765 words, 4 images.

\title{
Light-Directed Writing of Chemically Tunable Narrow-Band Holographic Sensors
}

Ali K. Yetisen, * Haider Butt, Fernando C. Vasconcellos, Yunuen Montelongo, Colin A. B. Davidson, Jeff Blyth, Leon Chan, J. Bryan Carmody, Silvia Vignolini, Jeremy Baumberg, Ullrich Steiner, Timothy D. Wilkinson, and Christopher R. Lowe

A. K. Yetisen, Dr. F. C. Vasconcellos, Dr. C. A. B. Davidson, Dr. J. Blyth, L. Chan, and Prof. C. R. Lowe

Department of Chemical Engineering and Biotechnology, University of Cambridge, Tennis

Court Road, Cambridge, CB21QT, UK.

E-mail: ay283@cam.ac.uk

Dr. H. Butt, Y. Montelongo, and Prof. T. Wilkinson

Electrical Engineering Division, Department of Engineering, University of Cambridge,

Cambridge, CB3 0FA, UK.

Dr. S. Vignolini, Prof. U. Steiner, and Prof. J. Baumberg

Cavendish Laboratory Department of Physics University of Cambridge J. J. Thomson Avenue, Cambridge, CB3 OHE, UK.

Dr. J. B. Carmody

The Children's Hospital of the King's Daughters, Norfolk, 23507, VA, USA.

Keywords: optical sensors, holography, lasers, hydrogels, nanoparticles

Dynamic photonic structures can be tuned by changing periodic structures and/or the index of refraction. ${ }^{[1]}$ These dynamic photonic structures enable optically responsive capability to control the properties of light and act as optical transducers to sense external stimuli. ${ }^{[2]}$ The tunable optical sensors in the visible and near-infrared region offer great promise for designing adaptive optical materials, telecommunication devices and sensors. Such sensors have been prepared by various methods, including microfabrication, self-assembly or a combination of both. ${ }^{[3]}$ However, achieving narrow-band response with a wide-tunability range to construct off-axis optical sensors still remains a significant challenge.

Recently, we developed a fundamentally distinct optical sensing platform based on Denisyuk reflection holography, ${ }^{[4]}$ in which laser light is used to organize nanoparticles (NPs) in a fraction of time, cost and complexity compared to previous approaches. ${ }^{[5]}$ This allows the 
fabrication of holographic sensors that display improved versatility and scalability. This platform utilizes an efficient approach to produce off-axis chemical-stimuli responsive holographic sensors with a large, reversible narrow-band tunability, using metallic nanoparticles that can be organized in density-concentrated 3D regions. Our sensor employs a simultaneous lateral and vertical periodic diffraction grating of silver nanoparticles dispersed within a poly(hydroxyethyl methacrylate)-based (pHEMA) matrix with a dry thickness of approximately $10 \mu \mathrm{m}$. The diffracted light is spectrally concentrated at a specific narrowband color due to the vertically-ordered periodicity.

We use 6 ns-pulsed laser $(\lambda=532 \mathrm{~nm})$ standing waves to order the density of silver nanoparticles into regions with a periodicity of approximately half of the wavelength distributed throughout the cross section of the polymer matrix. The fabrication of the holographic sensors begins with UV-initiated free radical polymerization of the pHEMAbased hydrogel on a $\mathrm{O}_{2}$-plasma-treated poly(methyl methacrylate) (PMMA) substrate (Figure 1(a)). Subsequently, $\mathrm{Ag}^{+}$ions are perfused into the pHEMA polymer matrix (Figure 1(b)), and reduced with a photographic developer to form silver nanoparticles $\left(\mathrm{Ag}^{0}\right)$ (Figure 1(c)). The Ag nanoparticles within the hydrogel matrix are selectively and photochemically patterned at the areas where laser light constructively interferes to form a well-ordered photonic structure (Figure 1(d)) (see Supporting Information). The resulting hologram is instantaneously useable and it acts as a sensor by undergoing changes in diffraction properties during swelling and shrinking in the presence of analytes (Figure 1(e-f)). Our holographic sensor can be functionalized with, for example, methacrylic acid (MAA) that reversibly binds $\mathrm{H}^{+}$, and is therefore sensitive to $\mathrm{pH}$ variations. The deprotonation, and hence ionization, of carboxyl groups in MAA, increases osmotic pressure within the hydrogel. Consequently, this results in water uptake and swells the hydrogel. This swelling increases the spacing of nanoparticles in primarily the vertical (and to a lesser extent in lateral) directions, which 


\section{Submitted to MATERIALS \\ ADVANCED}

produces a dynamic narrow-band diffraction regime. As the concentration of $\mathrm{H}^{+}$decreases from $\mathrm{pH} 4.00$ to $\mathrm{pH} 8.00$ under physiological $(150 \mathrm{mM})$ ionic strengths, the wavelength of the peak originating from the structure systematically shifts from 495 to $815 \mathrm{~nm}$ (Figure 1(g)). The shift in the diffraction peak is visible to the naked eye throughout most of the measurement (Figure 1(h)). The process is reversible and the same sensor may be used for multiple analyses.

Apart from holographic sensing technology, several advances in $\mathrm{pH}$ sensing have been recently demonstrated. New electrochemical and field-effect transistor based sensors have utilized carbon fiber ${ }^{[6]}$ and carbon/metallic ${ }^{[7]}$ nanostructures for $\mathrm{pH}$ sensing, respectively. Notably, recent fluorescent sensors based on Förster resonance energy transfer using synthetic $\mathrm{DNA}^{[8]}$, genetically encoded red protein ${ }^{[9]}$ and antibody-conjugated $\mathrm{pH}$ dependent dye ${ }^{[10]}$ have been demonstrated for intracellular monitoring. Our holographic $\mathrm{pH}$ sensor offers unique attributes since it provides not only the interrogation and reporting transducer, but it also has the analyte-responsive hydrogel, rendering it label-free and reusable with remote sensing capability. Our flash photochemical process represents a first step towards multifunctional 3D hydrogel-based holographic sensors.

The response of the holographic sensor to $\mathrm{pH}$ change is rapid and reproducible. Consecutive swelling/shrinking steps were reproducible to within $\pm 3 \mathrm{~nm}$ over 20 successive buffer changes. No hysteresis was detected. For example, a pH change at 0.50 units from $\mathrm{pH}$ 5.50 to 6.00 took $\sim 1$ min to equilibrate, with a variation of peak diffraction wavelength of \pm 1 $\mathrm{nm}$ over three trials. The range of sensor response and the sensitivity of the hydrogel can be modulated by varying the concentration of the crosslinker and ionizable MAA in the polymer matrix, respectively. ${ }^{[11]}$ Such hydrogels can also be functionalized to be highly selective to a range of stimuli. ${ }^{[12]}$ 


\section{Submitted to \\ ADVANCED \\ MATERIALS}

In order to predict the interference patterns which produce the photonic structure in the hydrogel, we modelled our system during fabrication as arising from a superposition of different light waves. Hence, we evaluated the interference pattern created from three waves, including: (1) incident beam, (2) beam reflected from the mirror and (3) beam reflected internally at the hydrogel-water interface. Figure 2(a) illustrates a schematic of the laser light interference from the three beams.

We reconstructed the intensity distribution of the field along the hydrogel by simulating the interference of the three plane waves. Through computing the respective intensities and phases of individual plane waves, the resulting interference pattern was extracted. To visualize the intensity distribution in a $2 \mathrm{D}$ cross-section plane, the electromagnetic field at every point over an area of $10 \mu \mathrm{m} \times 10 \mu \mathrm{m}$ inside the hydrogel was evaluated. Figure 2(b) shows the result for the interference pattern of the three distinct plane waves, taking into account: angle, index of refraction, laser light wavelength, exponential decay of laser intensity while the laser light travels through the hydrogel-NP system and laser light phase changes upon reflection.

In order to simulate the laser-based photochemical patterning process, it is assumed that the energy of a single pulse gets transmitted instantaneously (6ns) to the particles before heat diffusion is involved. Notably, the localization of heat along the standing wave is essential to produce a well-defined photonic structure. The model was simplified by implying that photochemical patterning process occurs where the energy concentration exceeds a given threshold. Figure 2(c) shows the simulation of the resulting structure after photochemical patterning. White regions represent photochemically patterned material while black regions show non-patterned material. Along with the vertical standing wave $(\sim 193 \mathrm{~nm})$, a larger period wave $(\sim 3 \mu \mathrm{m})$ appears in the horizontal direction. The addition of the two standing waves results in this grating-like structure at the interface of the non-patterned region. The 
effect of laser wavelength, power, pulse duration, nanoparticle size and surface plasmon resonance on the mechanism of laser-based photochemical patterning and subsequent absorption by the nanoparticles has been widely discussed elsewhere. ${ }^{[13]}$ Studies have shown that the interaction between the laser light and the nanoparticles may influence particle diffusion, oxidation, structure and distribution. ${ }^{[14]}$ The outlined factors above could contribute to organizing the nanoparticle-polymer system which acts as a holographic sensor.

In order to characterize the spectral response of the underlying photonic structure, the holographic sensor was illuminated at normal incidence with a supercontinuum white light laser. The angle-dependent backscatter was recorded at different angles by a spectrophotometer positioned onto a goniometer. Figure 2(d) illustrates the spectrum for different collection angle and the intensity of the peak (in blue-to-red color scale). The grating structure diffracts light analogous to a grating coated with a multilayer. However, the diffraction is two orders of magnitude more efficient at $~ 532 \mathrm{~nm}$ compared with the other diffracted wavelengths (Figure 1(g)), due to the underlying grating multilayer structure parallel to the grazing surface. The combination of these two structures effectively provides an intense color-selective back-diffraction at angles away from the sample normal (see Supporting Information).

To understand the optical response of the multilayer structure, finite-element simulations were performed. The simulated model (Figure 3(a)) comprised of a cross section of six stacks composed of silver nanoparticles. Due to the limitations of computational software only the 1D multilayer structure was simulated and not the multilayer grating that produces the monocoloured diffraction. Therefore, the simulations only show the band gaps (regions of enhanced reflection) displayed by the multilayer structure and not the diffraction effects. The 1D periodic array of nanoparticle stacks was generated in MATLAB, with each stack consisting of 60 nanoparticles (generated using normal distribution with a mean diameter of $13 \pm 10 \mathrm{~nm}$ ) 
(See Supporting Information). Within each stack the particles were uniformly distributed in the vertical direction, while in horizontal direction, normal random distribution was used.

The spacing (vertical lattice constant) between stacks was set to $193 \mathrm{~nm}$ which is equal to $\lambda / 2 \mathrm{n} \cos \theta\left(\theta=5^{\circ}\right)$, where $\lambda=532 \mathrm{~nm}$ is the wavelength of laser used for photochemical patterning process and $n=1.37 \pm 0.03$ is the index of refraction of the fully swollen hydrogel. The effective index of refraction of the non-patterned hydrogel was $1.46 \pm 0.01$ and it decreased to $1.43 \pm 0.01$ after photochemical patterning of nanoparticles (see Supporting Information). Within the hydrogel, the laser wavelength of $532 \mathrm{~nm}$ is reduced to approximately $386 \mathrm{~nm}$, and hence a vertical periodic structure with a lattice constant of around $193 \mathrm{~nm}$ is produced. The model was used to simulate the wave propagation through the lattice for the wavelength range of $400 \mathrm{~nm}$ to $1000 \mathrm{~nm}$. Figure 3(c) illustrates the simulated reflection spectrum from the multilayer structure. The spectrum shows peak reflectivity at $536 \mathrm{~nm}$, the wave propagation of which is shown in Figure 3(a). This is the wavelength that defines the green color of the holographic sensor, and shows that the color of the sensor is dictated by the spacing between the nanoparticle stacks. The simulation was also repeated with half the number of nanoparticles per stack, as shown in Figure 3(b). Decreasing the number of nanoparticles decreases the effective refractive index of the stacks, and therefore this trend reduces the index contrast between stacks and the hydrogel. This leads to a weaker reflection band gap, which is clearly observed from the simulated reflection spectra shown in Figure 3(c). The multilayer stack with 30 particles per stack displays about 55\% weaker band gap and reflects less light. The position of the reflection band does not change as the effective lattice constant remains unchanged.

Additional simulations were performed to analyze the effect of expanding the holographic sensor on the reflection band gaps. A multilayer structure with six nanoparticle stacks was modelled with the spacing between the stacks set to be $182 \mathrm{~nm}$. Figure 3(d) shows that the 
multilayer structure displayed a reflection-band gap centered at a wavelength of approximately $500 \mathrm{~nm}$ (the least transmitted). This is an equivalent of the optical response displayed by the holographic sensor at a pH of 4.00 (Figure 1(g)). To simulate the dynamic tunability, the multilayer structure was expanded while keeping the number and dimensions of the nanoparticles the same. As shown in Figure 3(d-f), the expansion results in an increase in the effective-stack spacing, stack size and a reduction in the concentration of nanoparticles per stack. The overall effect of these changes on the wave propagation is clearly observed in the simulated reflection spectra. The reflection spectra (Figure 3(g)) show a red shift in the reflection bands with the increasing stack spacing. Figures 3(d-f) show the multilayer geometry for fringe spacings of $182 \mathrm{~nm}, 237 \mathrm{~nm}$ and $297 \mathrm{~nm}$. However, the reflection spectrum simulations were also performed for stack spacings of $193 \mathrm{~nm}, 211 \mathrm{~nm}, 268 \mathrm{~nm}$ and $280 \mathrm{~nm}$, as displayed in Figure 3(g). The expanding multilayer structure displays a changing color (reflection band) varying across the visible spectrum from approximately $500 \mathrm{~nm}$ to 815 $\mathrm{nm}$. It is also observed that with an increase in stack spacing, the efficiency of the multilayer structure decreases, shown by the decrease in the intensity of the reflection band. This is due to the decrease in the concentration of nanoparticles present in each stack, which reduces the effective index contrast between the nanoparticle stacks and medium. Another contributing factor is that the scattering strength of each Ag NP increases at Mie plasmon resonances in the blue/green region, so the total amount of scattering decreases as the Bragg resonance shifts to longer wavelengths. While Figure 3(g) shows that the wavelength-shift trend expected from the model is reproduced experimentally with regard to a fall in efficiency at higher wavelengths, the peak broadening predicted was not observed. It is notable that the simulated reflection peaks are considerably wider than found experimentally. This is likely to be due to the limited number of periods of the lattice which were modelled (computational limitations do not permit us to model the full $10 \mu \mathrm{m}$ depth of the hologram). 
To demonstrate the utility of our sensor, we describe a clinical application to analyze acidbase balance in artificial urine samples ${ }^{[15]}$ (see Supporting Information). Urine $\mathrm{pH}$ in a healthy individual is in the range of 5.0-9.0. ${ }^{[16]}$ Analysis of urine $\mathrm{pH}$ has diagnostic utility in, for example, the evaluation of urinary tract infections (UTIs) ${ }^{[17]}$ and renal tubular acidosis. ${ }^{[18]}$ Modification of the urine $\mathrm{pH}$ is a key element of treatment for certain types of kidney stones, ${ }^{[19]}$ or toxic ingestions. ${ }^{[20]}$ We have tailored the tunability range of our holographic sensors to measure the $\mathrm{pH}$ from 4.5-7.0 in artificial urine samples. Figure 4(a) shows the holographic sensor's wavelength shift as a function of $\mathrm{pH}$ in the physiological range. The sensor has exceptional sensitivity from $\mathrm{pH}$ 4.6-6.6. This wavelength range can be tuned anywhere from UV to near infrared. Figure 4(b) presents the corresponding colorimetric response.

We have developed a viable platform technology to fabricate tunable holographic sensors via laser light. We have reported the use of a laser pulse (6 ns) to organize an off-axis 3D tunable sensor consisting of well-ordered silver nanoparticles embedded within hydrogel matrix. The holographic sensor can be finely modulated to diffract narrow-band light based on changes in nanoparticle spacing and index of refraction. Our work demonstrates that holographic sensors display large reversible band gap tunability in response to variation in $\mathrm{pH}$. These holographic sensors diffract light from the visible region to the near-infrared region $\left(\lambda_{\text {peak }} \approx 495-815 \mathrm{~nm}\right)$. The clinical application of our holographic sensors was demonstrated by $\mathrm{pH}$ sensing of artificial urine over the physiological range (4.5-9.0), with exceptional sensitivity between $\mathrm{pH} 5.0$ and 6.0.

Our method allows the use of a diverse array of substrate matrices, ranging from synthetic to natural polymers. The diffraction angle and holographic pattern within the matrix can also be controlled depending on the desired application. Other metallic nanoparticles and dyes can also be used in building well-ordered diffraction gratings with our method. Our sensing 


\section{Submitted to \\ ADVANCED \\ MATERIALS}

platform has extremely attractive attributes such as simple fabrication without requiring cleanroom facilities, flexible characteristics desirable for printing, and overall low-cost with potential scalability. We anticipate that our platform strategy of fabricating holographic sensors with wide optical ranges will lead to many novel applications from printable diffraction grating devices to rapid colorimetric sensors and biomedical sensing.

\section{Experimental Section}

Materials and Instruments: 2-Hydroxyethyl methacrylate (HEMA) (ultrapure 99+\%), ethylene glycol dimethacrylate (EDMA) (98\%), methacrylic acid (MAA) (99\%), 2,2dimethoxy-2-phenylacetophenone (DMPA) (99\%), silver nitrate (99\%), citric acid (99.5\%), sodium phosphate dibasic $\left(\mathrm{Na}_{2} \mathrm{HPO}_{4}\right)(99.0 \%)$, L-ascorbic acid (99\%), sodium carbonate $(99.9 \%)$ and sodium hydroxide $(\geq 98.0 \%)$ were purchased from Sigma-Aldrich, U.K. 4methylaminophenol sulfate (99\%) was purchased from Acros Organics, U.K. Poly(methyl methacrylate) (PMMA) (0.5 mm thick) was purchased from Goodfellow Cambridge Ltd (Huntingdon, U.K.). Citric acid - $\mathrm{Na}_{2} \mathrm{HPO}_{4}$ buffer solutions (150 mM) were used to obtain desired $\mathrm{pH}$ values.

Femto plasma cleaner (Diener electronic, Ebhausen, Germany) was used under 1 torr vacuum. Single-side aluminized polyester film was purchased from HiFi Industrial Film Ltd. (Stevenage, U.K.). Stratalinker $2400 \mathrm{UV}$ Crosslinker $(\sim 350 \mathrm{~nm}, 4000 \mu \mathrm{watts} / \mathrm{cm} 2)$ was purchased from RS Components (Corby, U.K.). Nd-Yttrium-Aluminum-Garnet pulsed laser (high power compact Q-switched Nd:YAG oscillator with super gaussian resonator, $700 \mathrm{~mJ}$ @ 1064 nm, $10 \mathrm{~Hz}$ ) with a second harmonic generator, 350 mJ @ 532 nm 10 Hz, thermally stabilized with wavelength separation) and supercontinuum white light laser were purchased from Lambda Photometrics (Harpenden, U.K.) and Fianium (SC400, Eugene, OR), 


\section{Submitted to \\ ADVANCED \\ MATERIALS}

respectively. AveSpec 2028 spectrophotometer, 2048-pixel InstaSpec IV CCD detector, QE6500 spectrometer and a bifurcated cable (FC UV 600-2, $600 \mu \mathrm{m}$ Fiber, $2 \mathrm{~m}$ length, SMA terminations) were purchased from Avantes (Apeldoorn, The Netherlands) and Ocean Optics (Dunedin, FL). FE20 FiveEasy pH meter was purchased from Mettler Toledo (Leicester, U.K). Abbe refractomer (Atago 4t) with an LED was purchased from Atago USA (Bellevue, WA). A plano-convex lens (laser grade PCX lens, $25 \mathrm{~mm}$ diameter x $75 \mathrm{~mm}$ FL, uncoated) was purchased from Edmund Optics Ltd. (York, U.K.). AvaSoft (v7.5) software, Igor Pro, MATLAB (v8.1) and COMSOL Multiphysics (v4.3b) were used for data processing and simulations. Lumix DMC-FZ20 camera was used for imaging. TEM images were obtained on a FEI Tecnai G2 (Oregon, USA) operated at $120 \mathrm{kv}$. Images were recorded with an AMT 60B camera running Deben software.

Preparation of Holographic Sensors: 91.5 mol\% HEMA, 2.5 mol\% EDMA, 6 mol\% MAA solution was mixed with equal volume of $2 \%$ (w:v) DMPA in isopropanol, cast and polymerized onto PMMA substrates previously treated with $\mathrm{O}_{2}$ plasma. The hydrogel thickness was controlled to be approximately $10 \mu \mathrm{m}$. Ag nanoparticles $(\sim 10-100 \mathrm{~nm}$ in diameter) were prepared through the incorporation and reduction of aqueous $\mathrm{AgNO}_{3}(1 \mathrm{M})$ within the hydrogel. Well-ordered $\mathrm{Ag}$ nanoparticle structures within the hydrogel were formed via a single 6-ns pulse of laser beam directed at the sample elevated at $5^{\circ}$ from the normal, backed by a mirror and immersed in aqueous solution at pH 4.0 (see Supplementary Information).

\section{Supporting Information}

Supporting Information is available online from the Wiley Online Library or from the author.

\section{Acknowledgements}




\section{ADVANCED \\ Submitted to \\ MATERIALS}

We thank Michael Monteiro and Caterina Ducati for useful discussions. F.C.V. would like to

thank the Postdoctoral Fellowships from FAPESP (Grant No. 2011/06906-6) and CNPq

(Grant No. 209869/2013-5).

Received: ((will be filled in by the editorial staff))

Revised: ((will be filled in by the editorial staff))

Published online: ((will be filled in by the editorial staff))

Insert references here 


\section{ADVANCED \\ Submitted to \\ MATERIALS}

a

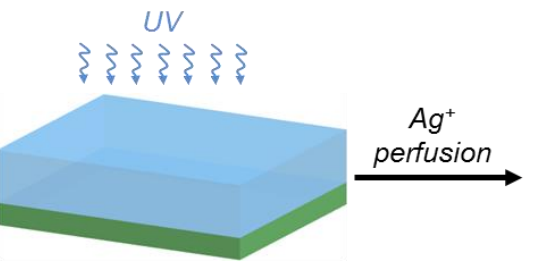

f

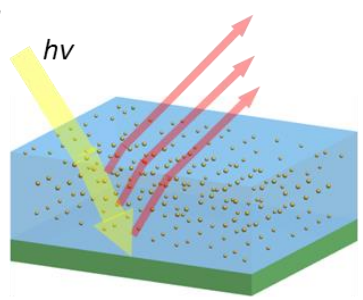

b

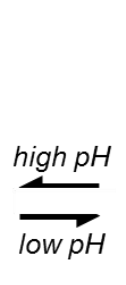

$\mathbf{e}_{h V}$

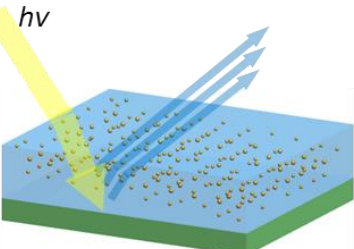

Nanoparticle

formation

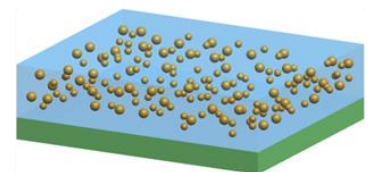

d $\begin{gathered}\text { Laser } \\ \text { irradiation }\end{gathered}$

$\lambda=$ $532 \mathrm{~nm}$

Analyte

response

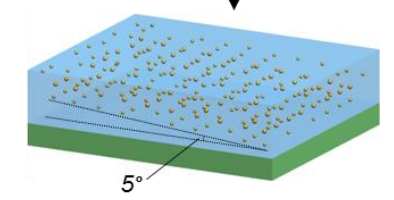

g

g $\quad \mathrm{pH}$

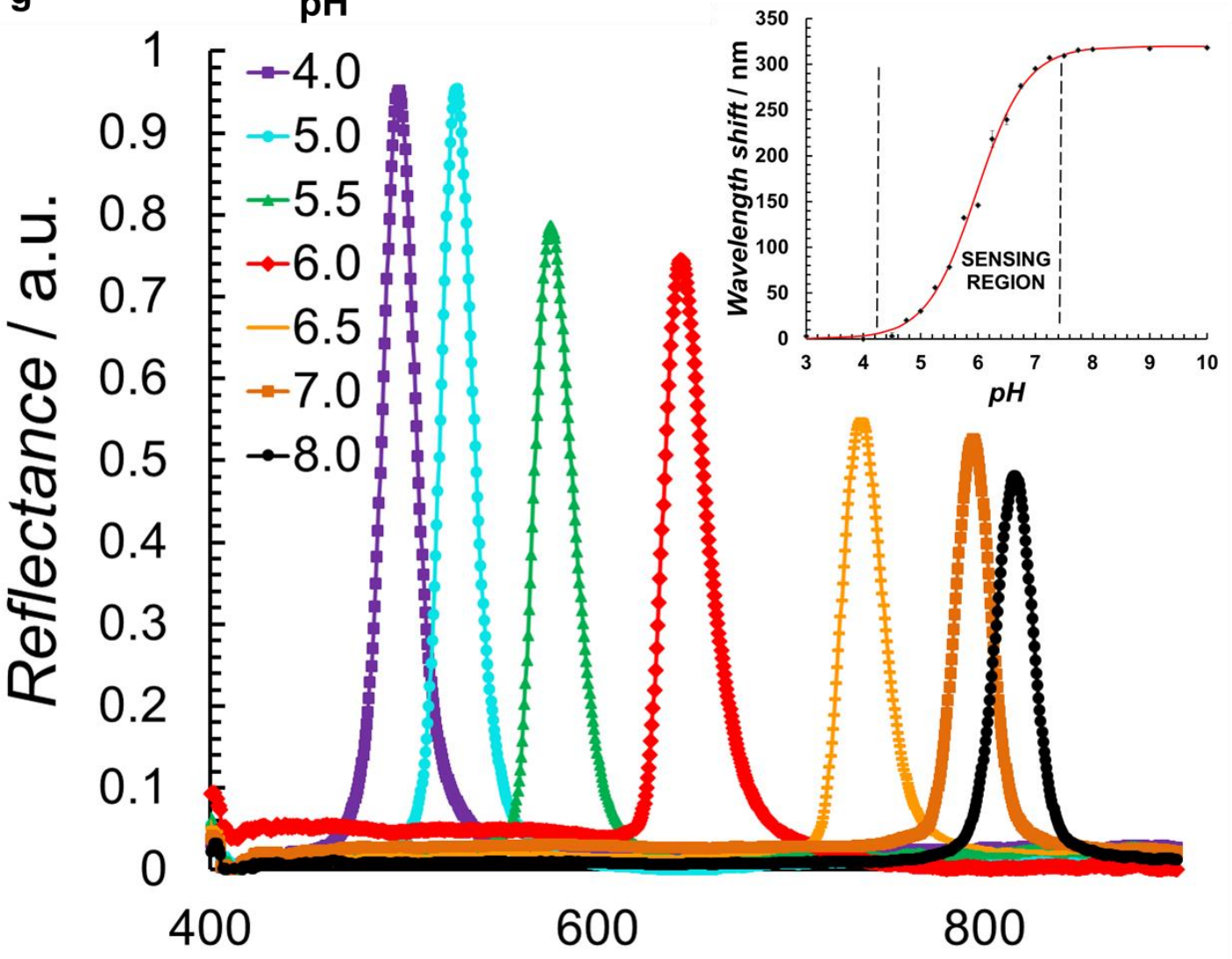

Wavelength / nm

h

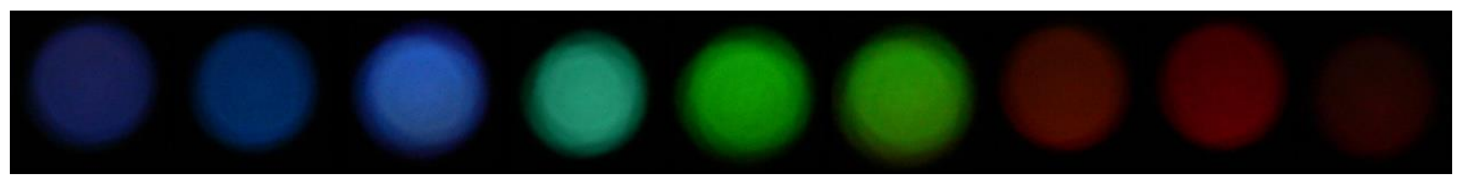

$\begin{array}{llllllllll}\text { pH } & 4.00 & 4.50 & 4.75 & 5.00 & 5.25 & 5.50 & 5.75 & 6.00 & 6.25\end{array}$

Figure 1. Fabrication holographic sensors and its tuning mechanism. (a) HEMA monomers functionalized with carboxyl groups were polymerized on an $\mathrm{O}_{2}$-plasma-treated PMMA substrate. (b) $\mathrm{Ag}^{+}$ions were perfused into pHEMA hydrogel film. (c) $\mathrm{Ag}^{+}$ions were reduced 


\section{Submitted to \\ ADVANCED}

to metallic $\mathrm{Ag}^{0}$ nanoparticles in the pHEMA hydrogel film using a photographic developer.

(d) A holographic sensor was formed by photochemically patterning pHEMA-Ag nanoparticle system via a single $6 \mathrm{~ns} \mathrm{Nd}$ :YAG pulsed laser beam, elevated at $5^{\circ}$ from the normal and backed by a mirror (e) Swelling/shrinking of the holographic sensor by a reagent modulates both the domain spacing and the refractive-index contrast, and systematically shifts the diffracted wavelengths of light $(h v)$ from 500 to (f) $815 \mathrm{~nm}$ when the hydrogel expands in the direction normal to underlying substrate. (g) Tuning a holographic sensor by $\mathrm{pH}$ variation. Visible-near-infrared diffraction spectra of a holographic sensor swollen by different $\mathrm{pH}$ values using phosphate buffers. The largest wavelength shift is at $815 \mathrm{~nm}$ and the smallest is at $495 \mathrm{~nm}$ - a change of $165 \%$. The apparent pKa value was calculated as 5.98 using the Henderson-Hasselbalch equation (see Supporting Information). (h) Photographs of the $\mathrm{pH}$ responsive holographic film immersed in phosphate buffers of $\mathrm{pH} 4.00-6.25$. The images were taken under white light illumination. 


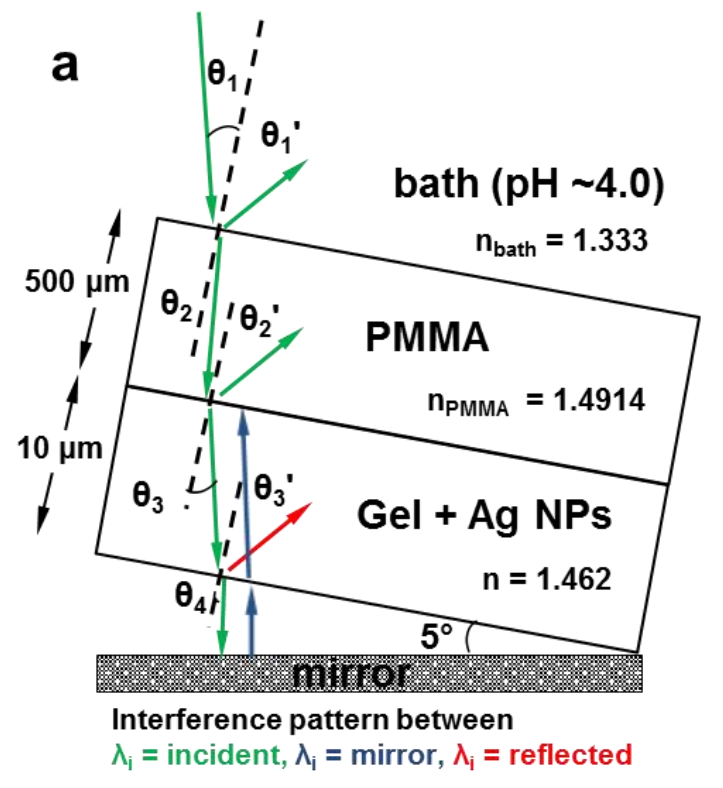

b

Submitted to MATERIALS
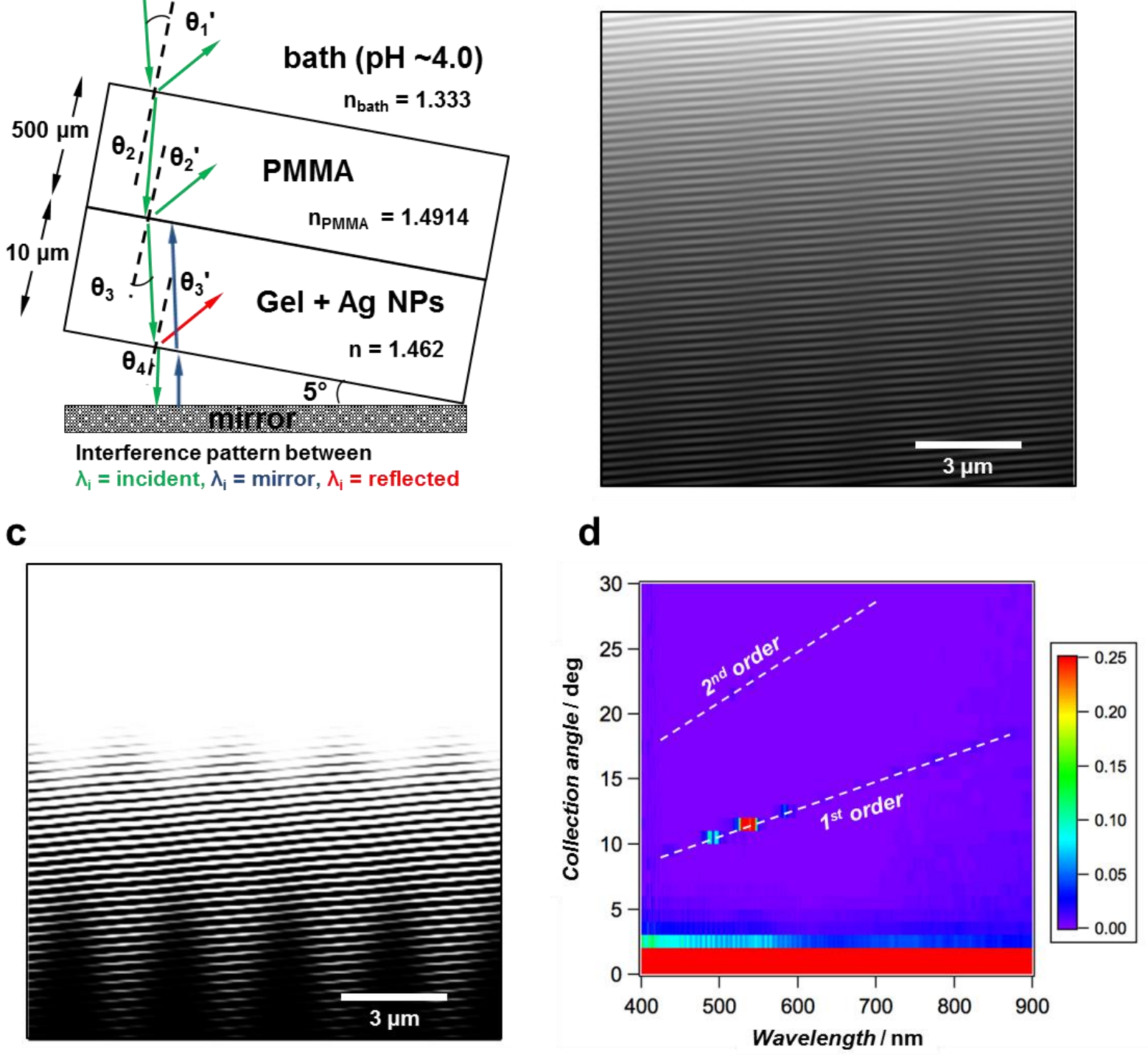

Figure 2. Photochemical patterning process based on the interference of multiple beams forms a well-ordered intensity field distribution. (a) Schematic of the laser-based photochemical patterning setup for the preparation of holograms, (b) Schematic of laser light interference beams inside the hydrogel, created from three beams: (1) incident beam, (2) beam reflected from mirror and (3) beam reflected internally at the hydrogel-water interface, (c) Intensity field distribution obtained from a holographic sensor with a tilting angle of $5^{\circ}$. (d) Angular resolved measurements from the sample. The zero order of diffraction $\left(0^{\circ}\right)$ is saturated to highlight the presence of the first and second diffracted orders. Using the grating 
equation and measuring the position of the order from the data, we estimate that the grating has a spacing of $3.01 \mu \mathrm{m}$.

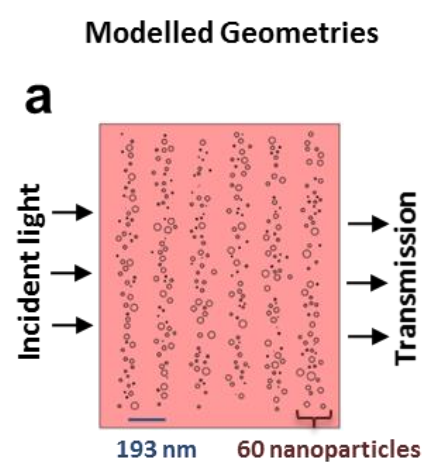

b

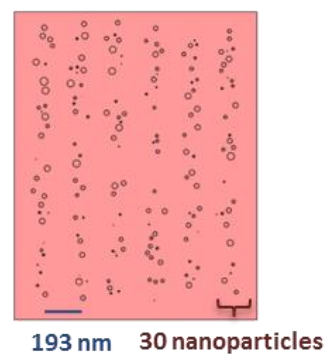

C

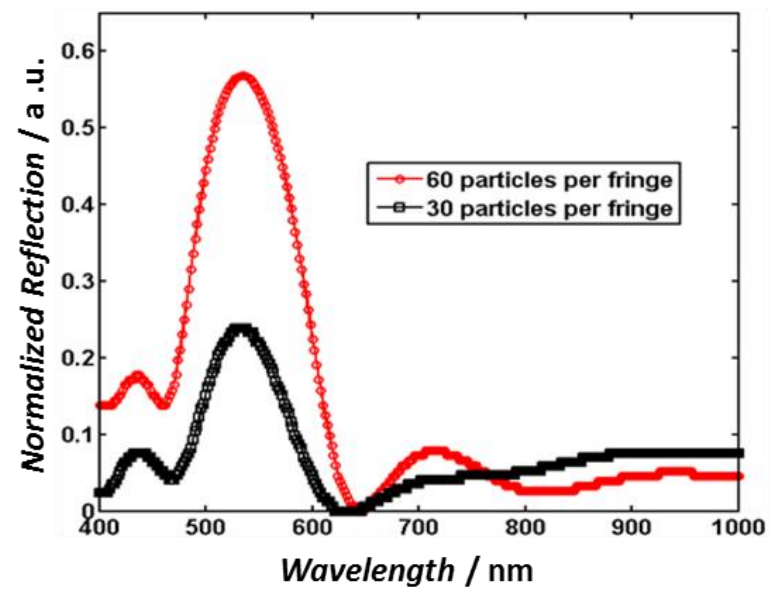

Modelled Geometries

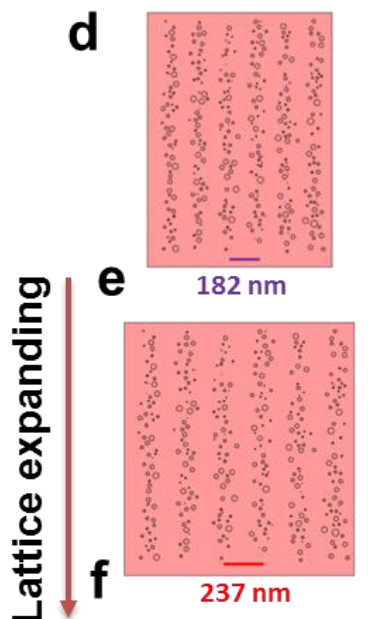

g

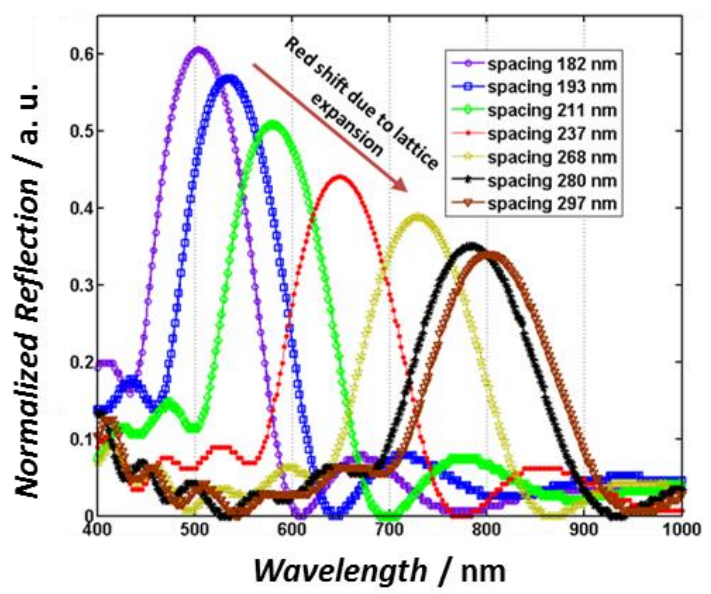

Figure 3. Simulated results for the reflection bands displayed by the holographic sensors. Simulated model geometry of the holographic sensor with a lattice constant of $193 \mathrm{~nm}$ containing (a) 60 and (b) 30 nanoparticles per fringe. (c) The computed reflection spectra for the two multilayer structures for (a) and (b). (d, e, f) Simulations of the geometries of the horizontally expanding multilayer structure and the respective wave propagation diagrams of 
the most reflected wavelength. $(\mathrm{g})$ The simulated reflection spectra for the multilayer structure with fringe spacing expanding from $182 \mathrm{~nm}$ to $297 \mathrm{~nm}$. In response to the expansion, the reflection band undergoes a red shift from $500 \mathrm{~nm}$ to $815 \mathrm{~nm}$ wavelengths.

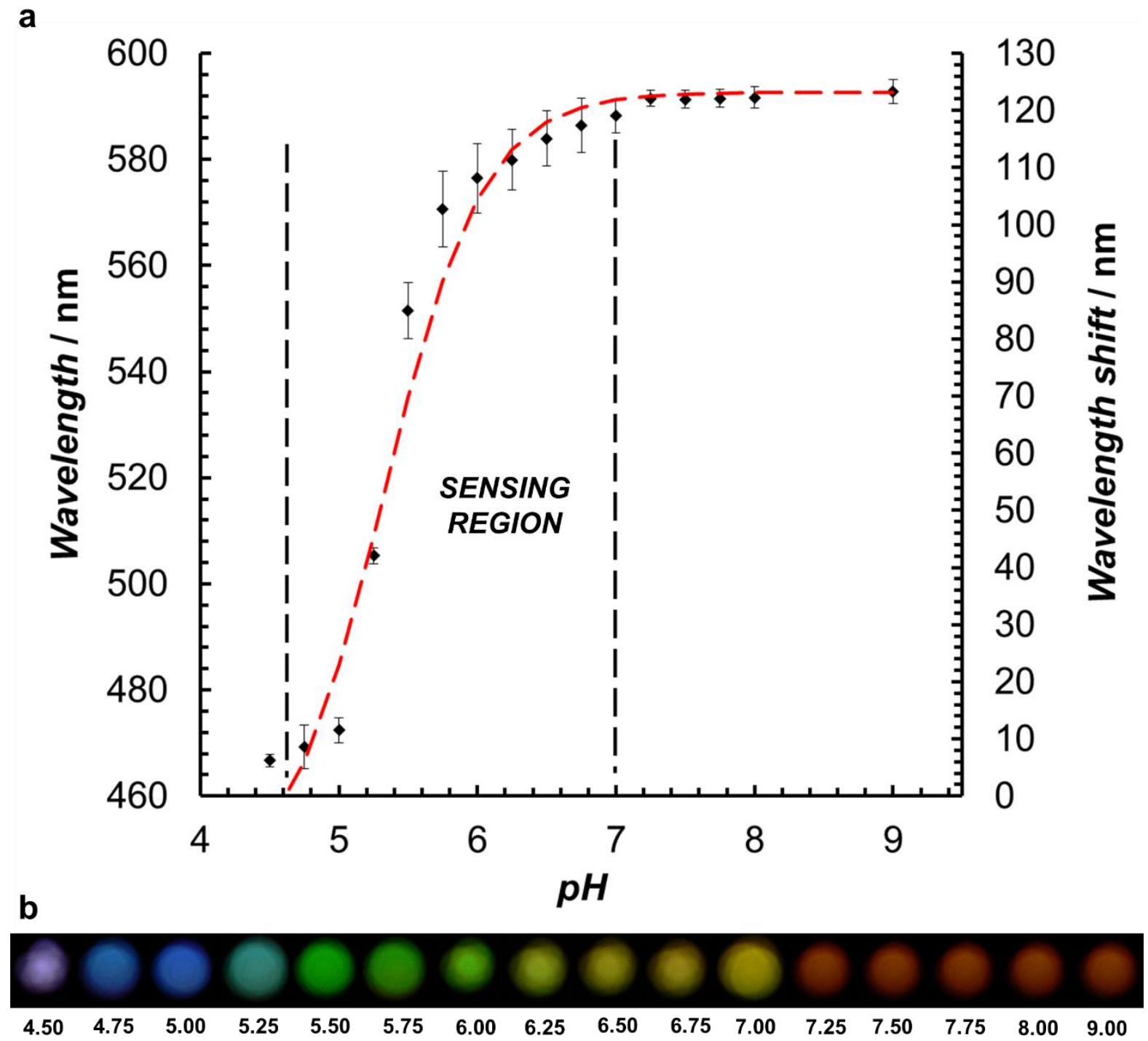

Figure 4. (a) Wavelength shift of a holographic sensor swollen by contact with different $\mathrm{pH}$ of artificial urine samples over the physiological range. Standard error bars represent three independent samples. The apparent pKa value was calculated as 5.20 using the HendersonHasselbalch equation. (b) Photographs of the pH-responsive holographic film sensor response to artificial urine samples ( $\mathrm{pH} 4.5-9.0)$. The images were taken under white light illumination. 


\section{ADVANCED \\ Submitted to \\ MATERIALS}

\section{The table of contents entry}

Tunable holographic sensor offers interrogation and reporting transducer as well as the analyte-responsive hydrogel, rendering it label-free and reusable. A single 6 ns laser pulse is used to fabricate holographic sensors consisting of silver nanoparticles embedded within a polymer film. The tunability of the sensor is demonstrated through $\mathrm{pH}$ sensing of artificial urine and validated through finite element simulations.

Keyword (see list)

Ali K. Yetisen, * Haider Butt, Fernando C. Vasconcellos, Yunuen Montelongo, Colin A. B. Davidson, Jeff Blyth, Leon Chan, J. Bryan Carmody, Silvia Vignolini, Jeremy Baumberg, Ullrich Steiner, Timothy D. Wilkinson, and Christopher R. Lowe

\section{Light-directed Writing of Chemically Tunable Narrow-Band Holographic Sensors}

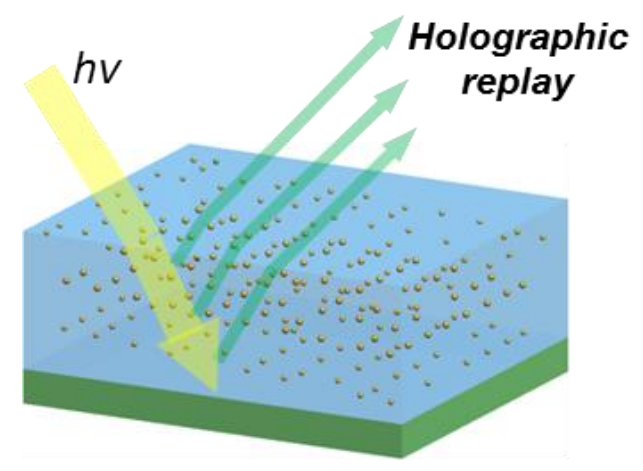


Copyright WILEY-VCH Verlag GmbH \& Co. KGaA, 69469 Weinheim, Germany, 2013.

\section{Supporting Information}

for Adv. Optical Mater., DOI: 10.1002/adom.((please add manuscript number))

\section{Light-directed Writing of Chemically Tunable Narrow-Band Holographic Sensors}

Ali K. Yetisen,* Haider Butt, Fernando C. Vasconcellos, Yunuen Montelongo, Colin A. B. Davidson, Jeff Blyth, Leon Chan, J. Bryan Carmody, Silvia Vignolini, Jeremy Baumberg, Ullrich Steiner, Timothy D. Wilkinson, and Christopher R. Lowe

\section{Holographic Sensor Preparation}

Hydrogel films were prepared with a monomer solution consisting of 91.5 mol\% HEMA, 2.5 mol\% EDMA and 6 mol\% MAA. The solution was mixed in a ratio of 1:1 (v:v) with $2 \%$ (w:v) DMPA in propan-2-ol. $200 \mu 1$ of the resulting solution was pipetted as a lateral blob on an aluminum coated polyester sheet (aluminum side facing up). PMMA substrates were manufactured through a casting method which does not have birefringence, a requirement for our fabrication method. These substrates were $\mathrm{O}_{2}$ plasma treated under a vacuum of 1 torr for 3 min to render the PMMA surface hydrophilic (Fig. S1(a)). The PMMA substrates were positioned with the $\mathrm{O}_{2}$ plasma treated side facing down on top of the prepared hydrogel solution blob on the aluminum coated polyester sheet. Aluminum sheet-PMMA spacers, such as silica or latex beads with average diameters ranging from 5 to $20 \mu \mathrm{m}$ may be used to control the thickness of the polymer gel during curing. The samples were subsequently exposed to UV light for $30 \mathrm{~min}$ to initiate free radical polymerization and therefore, crosslinking of the hydrogel network (Fig. S1(b)). Room temperature curing and thermal polymerization can also be used.

Silver nitrate $\left(\mathrm{AgNO}_{3}\right)(1 \mathrm{M}, 200 \mu \mathrm{l})$ dissolved in deionized water was pipetted as an elongated blob onto a glass sheet. Alternatively, silver perchlorate solution may be used in methanol 50:50 (methanol: water). The PMMA substrate-hydrogel, polymer side down, was 


\section{Submitted to $M$ \\ ADVANCED \\ MATERIALS}

placed over the elongated blob of $\mathrm{AgNO}_{3}$ and the sample was incubated in situ for 3 min to allow perfusion of $\mathrm{AgNO}_{3}$ into the hydrogel film (Fig. S1(c)). The positively charged $\mathrm{Ag}^{+}$, which have affinity to polymer matrix, were reduced to silver nanoparticles by immersing the PMMA substrate-hydrogel in a photographic developer ( $\mathrm{pH} 13.0)$ comprised of $0.3 \%(w / v) 4-$ methylaminophenol sulfate, $2 \%(\mathrm{w} / \mathrm{v})$ ascorbic acid, $5 \%(\mathrm{w} / \mathrm{v})$ sodium carbonate and $1.5 \%$ (w/v) sodium hydroxide, during 30 seconds (Fig. S1(d)). In the developer, ascorbic acid is the main contributor to $\mathrm{Ag}$ nanoparticle formation by donating electrons to the $\mathrm{Ag}^{+}$; the molecular configuration of the ascorbic acid changes to dehydroascorbic acid after donating electrons. The hydrogel-Ag nanoparticle system was washed thoroughly with deionized water, prior to immersion in 5\% (v/v) acetic acid (pH 2.5) to neutralize the developer for 30 seconds (Fig. S1(e)). Finally, the sample was washed in water, dried, and stored at room temperature (Fig. S1(f)). The hydrogel acts as a supporting medium for the embedded silver nanoparticles, while also preventing nanoparticle agglomeration.

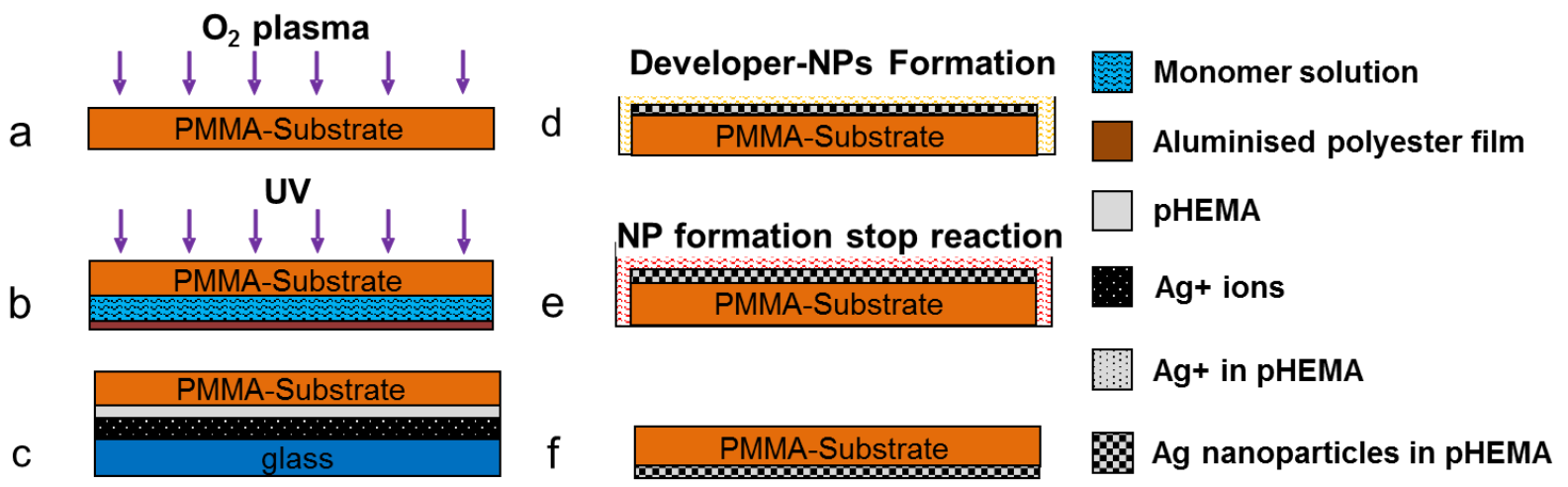

Figure S1. Cross-sectional schematics of the fabrication process of a holographic sensor. A PMMA slide was (a) treated with $\mathrm{O}_{2}$ plasma (b) placed treated $\mathrm{O}_{2}$ plasma side down on an elongated blob of monomer solution and exposed to UV $(\sim 350 \mathrm{~nm})$ to initiate free radical polymerization (c) placed polymer side down on an elongated blob of $\mathrm{AgNO}_{3}$ (d) immersed into photographic developer (e) placed in 5\% (v/v) acetic acid (f) washed with DI water and dried at room temperature. 


\section{ADVANCED \\ Submitted to \\ MATERIALS}

\section{Index of refraction measurements}

The index of refraction of the pure pHEMA is 1.51 [ref]. Abbe refractometer with an LED was used for the measurements. The effective index of refraction of the non-laser-patterned hydrogel (pHEMA, MAA, EDMA, Ag NPs) was $1.46 \pm 0.01$ and it decreased to $1.43 \pm 0.01$ after laser-based photochemical patterning of nanoparticles. Indirect measurement of the index of refraction of water-pHEMA system was $1.37 \pm 0.03$, achieved using a plano-convex lens. The focal points at air $(\mathrm{n}=1.00)$, water $(\mathrm{n}=1.33)$, ethanol $(\mathrm{n}=1.36)$ and decane $(\mathrm{n}=$ 1.41) were measured by placing the lens, convex side down, in a glass Petri dish, and submerging the lens into the target media. Next, the monomer mixture (91.5 mol\% HEMA, $2.5 \mathrm{~mol} \%$ EDMA and 6 mol\% MAA) was polymerized with a lens in the Petri dish, followed by the measurement of focal length of fully water swollen-hydrogel system. Finally, the index of refraction of water-hydrogel system was extrapolated based on the reference focal length measurements of air, water, ethanol and decane (Fig. S2).

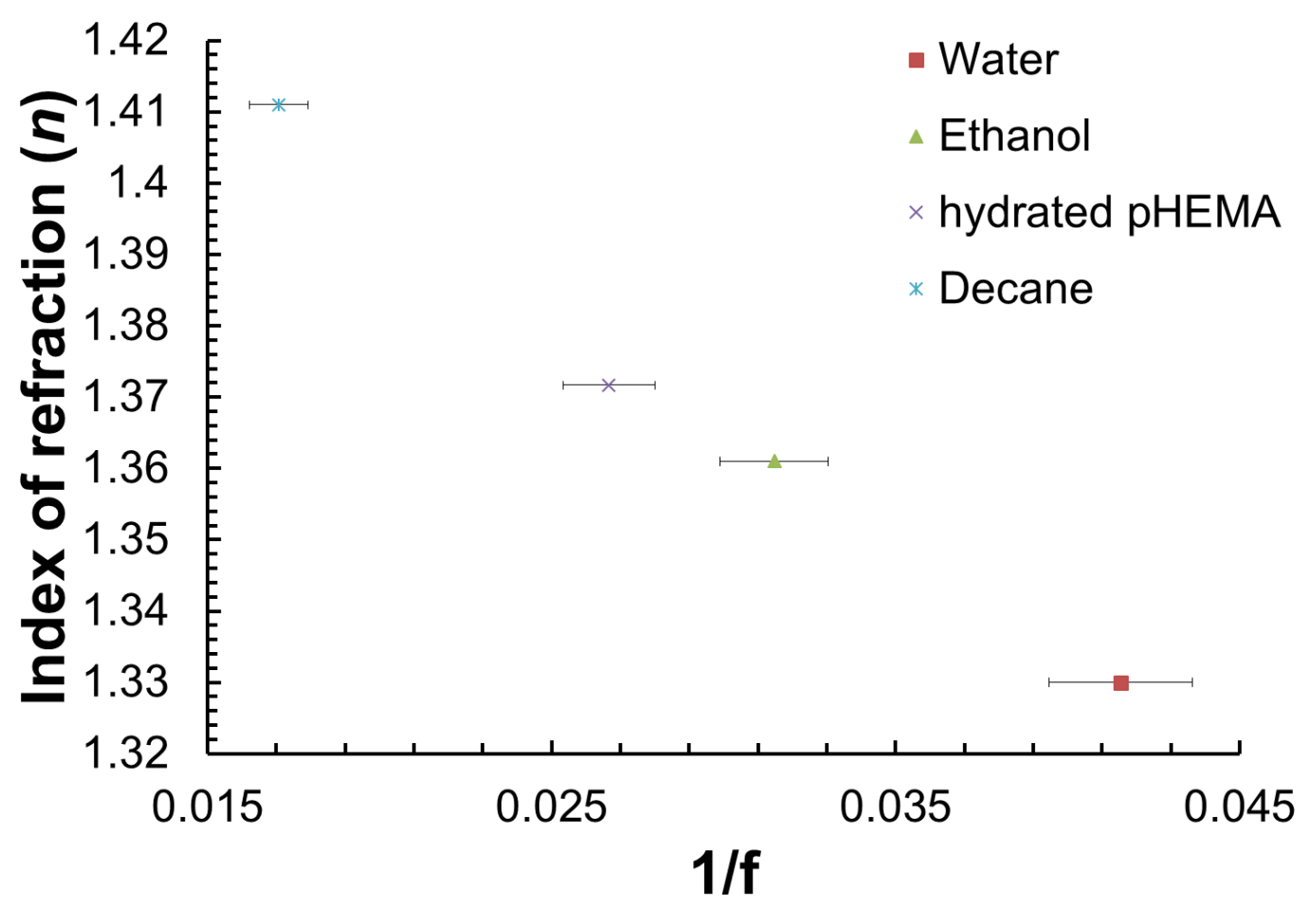


Figure S2. Estimation of index of refraction of the hydrated hydrogel (pHEMA, EDMA and MAA)

\section{Holographic sensor preparation via laser-based photochemical patterning process}

A levelled Petri dish, with a mirror positioned normal to the laser incidence beam in the bottom surface, was filled with phosphate buffer $(\mathrm{pH} 4.0)$. The prepared hydrogel-Ag nanoparticle system was photochemically patterned with via a single 6-ns pulse (Q-switch delay, $400 \mu \mathrm{s}$, a spot size of $1 \mathrm{~cm}$ in diameter) of $\mathrm{Nd}$ :YAG pulsed laser beam directed at the sample elevated at $5^{\circ}$ from the normal. Nd:YAG laser beam at $532 \mathrm{~nm}$ is redirected with dichroic mirrors as it passes through a spreader beam lens before it strikes onto the hydrogelAg nanoparticle system. The standing wave with a constructive interference pattern organizes the silver nanoparticles that eventually form a well-ordered photonic structure in the polymer matrix. The dynamic range of the tunable diffraction grating can be adjusted by varying the $\mathrm{pH}$ of the exposure bath. Ideally the refractive index of buffer should match the refractive index of the polymer to reduce the blazed grating effect. However, with commonly used buffers, this is not feasible. In addition, a photomask may be placed on the sample to create desirable patterns of holographic sensors. Additionally, concave and convex mirrors can also be used to obtain off-axis diffraction.

\section{Characterization of holographic sensors}

A holographic sensor fabricated in dry conditions (i.e. in air) was prepared. A supercontinuum white light laser was used for the illumination and a spectrophotometer positioned onto a goniometer was used for collecting the spectral response. Figure S3a illustrates the holographic diffraction in logarithmic scale. Figure S3b shows the hydrogel surface imaged using a microscope. 

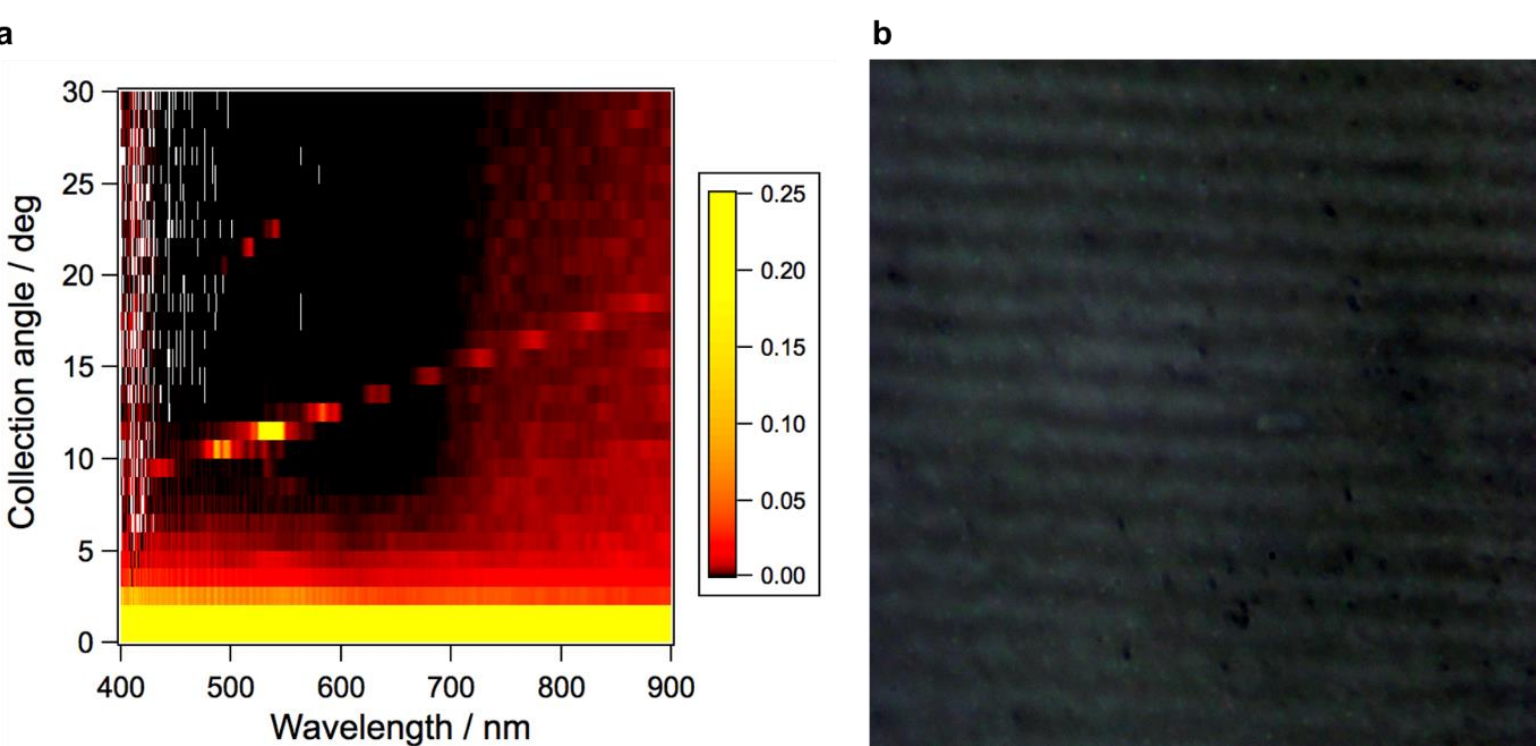

Figure S3. (a) Angular resolved measurements from the holographic sensors in logarithmic (b) Hydrogel surface images were captured using a microscope with a 100X objective.

\section{Spectral analysis of the holographic sensors}

Holographic sensors were cut to $0.5 \mathrm{~cm} \times 2.5 \mathrm{~cm}$ rectangles and spectroscopically analyzed in a $4 \mathrm{ml}$ disposable plastic cuvette containing different buffer and artificial urine reservoirs $(\mathrm{pH}$ 4.00-9.00). The solutions in the cuvette were kept at constant room temperature. The holograms were allowed to reach constant diffraction wavelength before a reading was taken.

\section{TEM analysis}

Hydrogels were released from their substrates with a razor blade and transferred to dry ethanol. This was replaced with 2 changes of acetonitrile for ten minutes each. They were transferred from that into a 50/50 mixture of acetonitrile and Araldite epoxy resin. The acetonitrile was allowed to evaporate overnight. The hydrogels were transferred through 3 changes of araldite for 2 hours each and the resin was cured at 60 degrees $\mathrm{C}$ for 48 hours. Vertical sections through the hydrogel were cut with a diamond knife (Deyemond, Germany) using a Leica Ultracut UCT (Leica, Vienna). They were mounted on 400 mesh copper grids 
and viewed in a FEI Tecnai G2 (Oregon, USA) operated at 120kv. Images were recorded with an AMT 60B camera running Deben software.

\section{Nanoparticle Size}

Nanoparticle size distribution before (Fig. S4(a)) and after laser-based photochemical patterning process (Fig. S4(b)) was measured via analyzing TEM images. ImageJ (National Institutes of Health) was used to analyze the images. Standard deviation values for the diameter of the nanoparticles before and after laser-based photochemical patterning process were $26 \pm 23 \mathrm{~nm}$ (137 nanoparticles) and $13 \pm 10 \mathrm{~nm}$ (2249 nanoparticles), respectively.

\section{a}

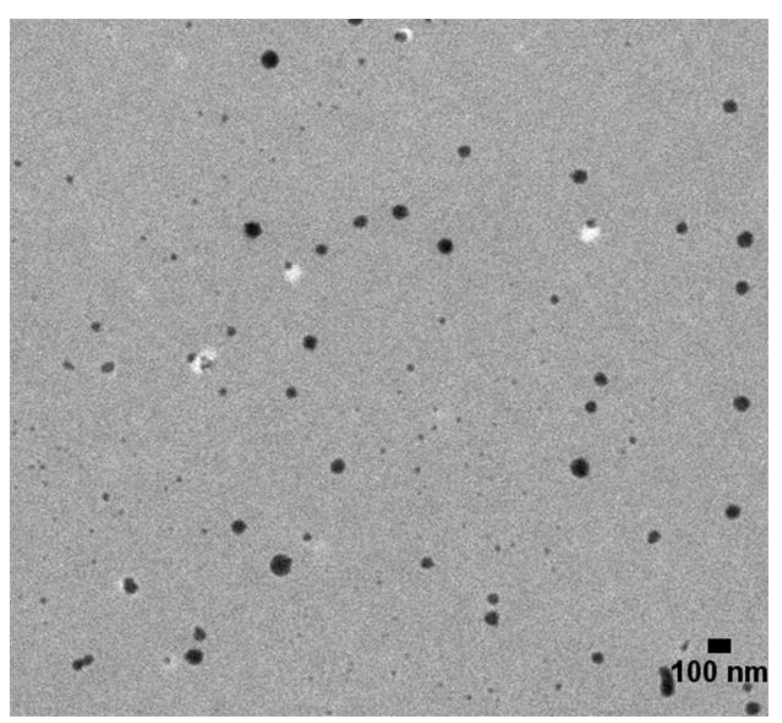

b

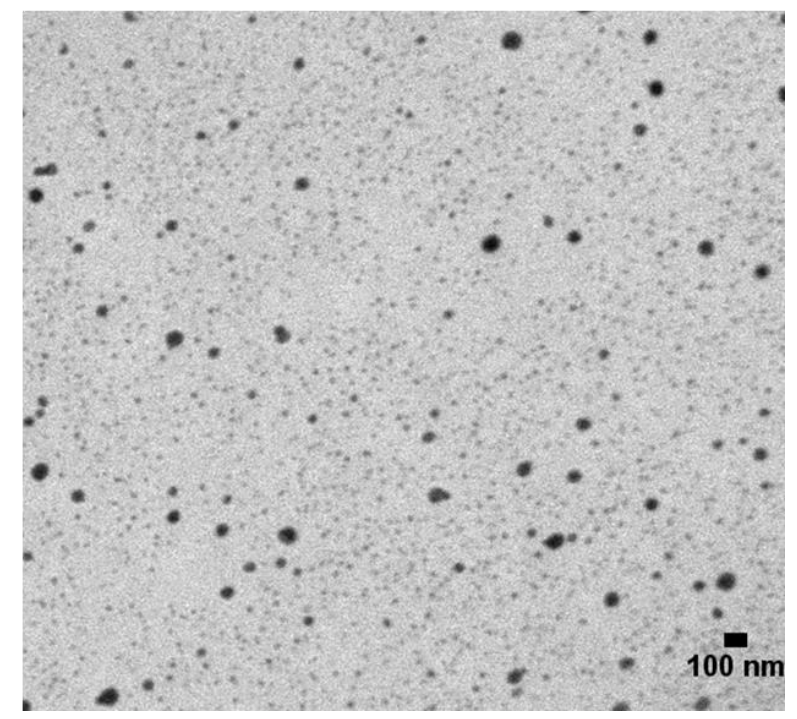

Figure S4. TEM images of the cross section of the Polymer + NP system (a) before (b) after laser-based photochemical patterning

\section{Artificial Urine: Preparation}

Artificial urine was prepared as described previously [Martinez et al., 2008]. pH was adjusted to the desired values using 1M hydrochloric acid and 1M sodium hydroxide (Table S1). 
Table S1. Composition of artificial urine

\begin{tabular}{|c|c|c|}
\hline Component & Quantity [g] & Concentration $\left[\mathrm{mmol} \mathrm{l}^{-1}\right]$ \\
\hline Peptone L37 & 1 & N/A \\
\hline Yeast extract & 0.005 & N/A \\
\hline Lactic acid & 0.1 & 1.1 \\
\hline Citric acid & 0.4 & 2 \\
\hline Sodium bicarbonate & 2.1 & 25 \\
\hline Urea & 10 & 170 \\
\hline Uric acid & 0.07 & 0.4 \\
\hline Creatinine & 0.8 & 7 \\
\hline Calcium Chloride. $2 \mathrm{H}_{2} \mathrm{O}$ & 0.37 & 2.5 \\
\hline Sodium Chloride & 5.2 & 90 \\
\hline Iron II sulfate. $7 \mathrm{H}_{2} \mathrm{O}$ & 0.0012 & 0.005 \\
\hline Magnesium sulfate. $7 \mathrm{H}_{2} \mathrm{O}$ & 0.49 & 2 \\
\hline Sodium sulfate. $10 \mathrm{H}_{2} \mathrm{O}$ & 3.2 & 10 \\
\hline Potassium dihydrogen phosphate & 0.95 & 7 \\
\hline Dipotassium hydrogen phosphate & 1.2 & 7 \\
\hline Ammonium chloride & 1.3 & 25 \\
\hline Distilled water & to $1 \mathrm{~L}$ & N/A \\
\hline Hydrochloric acid & to specific $\mathrm{pH}$ & 1 \\
\hline Sodium Hydroxide & to specific $\mathrm{pH}$ & 1 \\
\hline
\end{tabular}


Artificial Urine: Ionic strength

Ionic strength indicates the concentration of ions in a solution. Ionic strength is defined as

$I=\frac{1}{2} \sum_{i=1}^{n} c_{i} z_{i}^{2}=213.29 \mathrm{mmol} / \mathrm{L}$

where $c_{i}$ is the molar concentration of a specific ion in $\frac{m o l}{d m^{3}}$

$z_{i}$ indicates the charge of a specific ion

It was assumed that there was no ionic contribution made from peptone L37, yeast extract, urea, and creatinine.

\section{pKa value estimation}

A modified Henderson-Hasselbalch equation was used to determine the apparent pKa values.

$\lambda_{\text {shift }}=\lambda_{0}+\frac{\Delta \lambda}{\left(10^{\left(\mathrm{pK}_{\mathrm{a}}-p H\right)}+1\right)}$

where $\lambda_{\text {shift }}=$ wavelength shift, $\lambda_{0}=$ initial wavelength, $\Delta \lambda=$ wavelength difference 
To:

The Editor,

Advanced Optical Materials

$02 / 09 / 2013$

Subject: Submission of "full paper", titled "Light-directed writing of chemically tunable narrow-band holographic sensors"

Dear Dr. Gregory,

I am writing to submit our manuscript entitled, "Light-directed writing of chemically tunable narrow-band holographic sensors" which we would like to submit for full paper in Advanced Optical Materials. Chemically-tunable optical sensors are promising tools to sense external stimuli. Current methods to fabricate optical sensors are limited due to high turnaround times, complexity in preparation, broadband response and labeling requirement. In our manuscript, we demonstrate a label-free and reuseable holographic sensing platform that has fastturnaround time (minutes) and flexible to prepare off-axis chemically-tunable sensors that can operate in the entire visible spectrum and near-infrared. To our knowledge, this is the first report demonstrating the dynamic simulations for the tunability of a sensor using finite element method. To demonstrate the utility of our sensors, we show the $\mathrm{pH}$ response in urine samples. We envision that our sensing platform will find applications in in vitro diagnostics and microfluidic biomedical and optical devices. Because the article provides a practical approach to fabricating optical sensors for clinical applications, we believe our findings would appeal to Advanced Optical Materials readers interested in optics, polymer science and biomedical research and diagnostics. We anticipate that our strategy to fabricate 'smart' sensors using light will enable other researchers to adopt our vision for the development of multiplexed label-free sensors.

This manuscript describes original work and is not under consideration by any other journal. All authors have approved the manuscript and its submission to Advanced Optical Materials. Thank you for receiving our manuscript and considering it for review. We look forward to hearing from you at your earliest convenience.

Sincerely,

Ali Yetisen

Department of Chemical Engineering and Biotechnology

University of Cambridge

Tennis Court Road

Cambridge

CB2 1QT

Tel: +44 (0) 7918927974

e-mail: ay283@cam.ac.uk 


\section{Submitted to

[1] aY. Kang, J. J. Walish, T. Gorishnyy, E. L. Thomas, Nature Materials 2007, 6, 957960; bH. Kim, J. Ge, J. Kim, S.-e. Choi, H. Lee, H. Lee, W. Park, Y. Yin, S. Kwon, Nat Photon 2009, 3, 534-540; cS. Kim, A. N. Mitropoulos, J. D. Spitzberg, H. Tao, D. L. Kaplan, F. G. Omenetto, Nat Photon 2012, 6, 818-823.

[2] aY. Y. Li, F. Cunin, J. R. Link, T. Gao, R. E. Betts, S. H. Reiver, V. Chin, S. N. Bhatia, M. J. Sailor, Science 2003, 299, 2045-2047; bJ. Kim, J. Yoon, R. C. Hayward, Nature materials 2010, 9, 159-164.

[3] aS. O. Kim, H. H. Solak, M. P. Stoykovich, N. J. Ferrier, J. J. De Pablo, P. F. Nealey, Nature 2003, 424, 411-414; bS. Ogawa, M. Imada, S. Yoshimoto, M. Okano, S. Noda, Science 2004, 305, 227-229; cM. Deubel, G. von Freymann, M. Wegener, S. Pereira, K. Busch, C. M. Soukoulis, Nature materials 2004, 3, 444-447; dM. Qi, E. Lidorikis, P. T. Rakich, S. G. Johnson, J. D. Joannopoulos, E. P. Ippen, H. I. Smith, Nature 2004, 429, 538-542; eA. C. Arsenault, T. J. Clark, G. von Freymann, L. Cademartiri, R. Sapienza, J. Bertolotti, E. Vekris, S. Wong, V. Kitaev, I. Manners, R. Z. Wang, S. John, D. Wiersma, G. a. Ozin, Nature Materials 2006, 5, 179-184; fK. Aoki, D. Guimard, M. Nishioka, M. Nomura, S. Iwamoto, Y. Arakawa, Nat Photon 2008, 2, 688-692; gJ. S. Skibina, R. Iliew, J. Bethge, M. Bock, D. Fischer, V. I. Beloglasov, R. Wedell, G. Steinmeyer, Nat Photon 2008, 2, 679-683; hS. A. Rinne, F. GarciaSantamaria, P. V. Braun, Nat Photon 2008, 2, 52-56; iS. Takahashi, K. Suzuki, M. Okano, M. Imada, T. Nakamori, Y. Ota, K. Ishizaki, S. Noda, Nature materials 2009, 8, 721-725; jK. Ishizaki, S. Noda, Nature 2009, 460, 367-370; kY. Fuchs, O. Soppera, A. G. Mayes, K. Haupt, Adv Mater 2013, 25, 566-570; 1A. Llordes, G. Garcia, J. Gazquez, D. J. Milliron, Nature 2013, 500, 323-326; mM. Kolle, A. Lethbridge, M. Kreysing, J. J. Baumberg, J. Aizenberg, P. Vukusic, Advanced Materials 2013, 25, 2239-2245; nY. Fuchs, S. Kunath, O. Soppera, K. Haupt, A. G. Mayes, Advanced Functional Materials 2013, n/a-n/a.

[4] S. A. Benton, V. M. Bove, in Holographic Imaging, John Wiley \& Sons, Inc., 2007, pp. 173-180.

[5] aJ. L. Martinez-Hurtado, C. A. Davidson, J. Blyth, C. R. Lowe, Langmuir 2010, 26, 15694-15699; bC. A. B. Davidson, J. Blyth, C. R. Lowe, WO, PCT/GB2009/051346, 2010.

[6] M. A. Makos, D. M. Omiatek, A. G. Ewing, M. L. Heien, Langmuir 2010, 26, 1038610391.

[7] B. R. Huang, T. C. Lin, Applied Physics Letters 2011, 99.

[8] S. Modi, M. G. Swetha, D. Goswami, G. D. Gupta, S. Mayor, Y. Krishnan, Nature Nanotechnology 2009, 4, 325-330.

[9] M. Tantama, Y. P. Hung, G. Yellen, Journal of the American Chemical Society 2011, 133, 10034-10037.

[10] A. Grover, B. F. Schmidt, R. D. Salter, S. C. Watkins, A. S. Waggoner, M. P. Bruchez, Angew Chem Int Edit 2012, 51, 4838-4842.

[11] A. J. Marshall, J. Blyth, C. a. B. Davidson, C. R. Lowe, Analytical chemistry 2003, 75, 4423-4431.

[12] aJ. D. Ehrick, S. K. Deo, T. W. Browning, L. G. Bachas, M. J. Madou, S. Daunert, Nature materials 2005, 4, 298-302; bA. Lendlein, H. Jiang, O. Junger, R. Langer, Nature 2005, 434, 879-882; cS. H. Um, J. B. Lee, N. Park, S. Y. Kwon, C. C. Umbach, D. Luo, Nat Mater 2006, 5, 797-801; dM. Ehrbar, R. Schoenmakers, E. H. Christen, M. Fussenegger, W. Weber, Nature materials 2008, 7, 800-804; eA. M. Kloxin, A. M. Kasko, C. N. Salinas, K. S. Anseth, Science 2009, 324, 59-63; fE. F. Banwell, E. S. Abelardo, D. J. Adams, M. a. Birchall, A. Corrigan, A. M. Donald, M. Kirkland, L. C. Serpell, M. F. Butler, D. N. Woolfson, Nature materials 2009, 8, 596-600; gM. A. C. 


\section{ADVANCED \\ Submitted to \\ MATERIALS}

Stuart, W. T. S. Huck, J. Genzer, M. Muller, C. Ober, M. Stamm, G. B. Sukhorukov, I. Szleifer, V. V. Tsukruk, M. Urban, F. Winnik, S. Zauscher, I. Luzinov, S. Minko, Nat Mater 2010, 9, 101-113.

[13] aA. Plech, V. Kotaidis, M. Lorenc, J. Boneberg, Nat Phys 2006, 2, 44-47; bD. Werner, S. Hashimoto, The Journal of Physical Chemistry C 2010, 5063-5072; cD. Werner, A. Furube, T. Okamoto, S. Hashimoto, J Phys Chem C 2011, 115, 8503-8512; dA. Kyrsting, P. M. Bendix, L. B. Oddershede, Nano Letters 2013, 13, 31-35.

[14] aP. M. Hansen, V. K. Bhatia, N. Harrit, L. Oddershede, Nano Letters 2005, 5, 1937 1942; bS. Barcikowski, A. Menendez-Manjon, B. Chichkov, M. Brikas, G. Raciukaitis, Applied Physics Letters 2007, 91; cL. Bosanac, T. Aabo, P. M. Bendix, L. B.

Oddershede, Nano Lett 2008, 8, 1486-1491; dD. C. Schinca, L. B. Scaffardi, F. A. Videla, G. A. Torchia, P. Moreno, L. Roso, J Phys D Appl Phys 2009, 42; eR. Saija, P. Denti, F. Borghese, O. M. Marago, M. A. Iati, Optics Express 2009, 17, 10231-10241; fV. Amendola, M. Meneghetti, Physical Chemistry Chemical Physics 2009, 11, 38053821.

[15] A. W. Martinez, S. T. Phillips, G. M. Whitesides, Proceedings of the National Academy of Sciences of the United States of America 2008, 105, 19606-19611.

[16] A. Kratz, M. Ferraro, P. M. Sluss, K. B. Lewandrowski, The New England Journal of Medicine 2004, 351, 1548-1563.

[17] J. A. Simerville, W. C. Maxted, J. J. Pahira, American Family Physician 2005, 71, 1153-1162.

[18] J. Rodríguez Soriano, Journal of the American Society of Nephrology 2002, 13, 21602170.

[19] aA. Hesse, D. Heimbach, World Journal of Urology 1999, 17, 308-315; bB. H. Eisner, D. S. Goldfarb, G. Pareek, The Urologic clinics of North America 2013, 40, 21-30.

[20] A. T. Proudfoot, E. P. Krenzelok, J. A. Vale, Journal of toxicology Clinical toxicology 2004, 42, 1-26. 